\title{
EVALUACIÓN DE LAS ESTRATEGIAS DE APRENDIZAJE EN LA UNIVERSIDAD: UN PROYECTO NECESARIO ANTE UN ALUMNADO DIVERSO
}

\section{EVALUATION OF LEARNING STRATEGIES IN COLLEGE: A NECESSARY PROJECT FOR A DIVERSE STUDENT BODY}

\author{
Lina Ma Tomás Pastor ${ }^{1}$ : Universidad Católica San Antonio de Murcia (España). \\ Itomas@ucam.edu
}

Sonia Ma Martínez Castro ${ }^{2}$ : Universidad Católica San Antonio de Murcia (España) martinez@ucam.edu

\author{
Ma José Lombarte Londres ${ }^{3}$ : Universidad Católica San Antonio de Murcia \\ (España). \\ milombarte@ucam.edu
}

\section{RESUMEN}

El presente artículo pretende reflejar cómo la gran diversidad del alumnado que presentan hoy las aulas universitarias españolas requieren del profesorado nuevas respuestas organizativas y metodológicas. Además de la gran riqueza que otorga esta diversidad descrita, se contempla también el desarrollo de una serie de dificultades en los estudiantes en habilidades básicas e instrumentales, no resueltas en etapas educativas anteriores, y que el profesorado universitario se encuentra ante sí. Se descubren mayores dificultades en habilidades lectoescritoras, un elevado índice de niveles de ansiedad, o más problemas a la hora del trabajo en equipo, cuestiones que se incorporan a las tareas del docente universitario hoy. Como respuesta a estos planteamientos, presentamos un proyecto de investigación basado en el estudio de distintos factores que influyen en el proceso de enseñanza aprendizaje, para detectar y abordar los problemas mencionados, proponiendo la tutoría personal en la Universidad como cauce adecuado para la puesta en práctica de este proyecto.

PALABRAS CLAVE: Diversidad - Dificultades de aprendizaje - Habilidades básicas- Estrategias - Docencia- Neuropsicología - Tutoría Universitaria.

\footnotetext{
${ }^{1}$ Lina Ma Tomás Pastor: Profesor Contratado Doctor: UCAM/Departamento de Educación/Murcia/España.

${ }^{2}$ Sonia Ma Martínez Castro: Profesor Contratado: UCAM/Departamento de Educación/Murcia/España.

${ }^{3} \mathbf{M}^{\mathbf{a}}$ José Lombarte Londres:Tutora Profesional: UCAM/Departamento de

Educación/Murcia/España.
} 


\section{Abstract}

This article aims to reflect how the great diversity of students, attending today at the classrooms in the Spanish university, require new organizational and methodological answers. In addition to the big wealth that gives the described diversity, also the development of a number of difficulties in students in basic and instrumental skills are observed, unresolved in earlier stages of education, and now found by university teachers. Greater difficulties at skills on read and writing, higher levels of anxiety and more problems in teamwork are discovered, which are added to the tasks of the Faculty today. In response to those approaches, we present a research project based on the study of different factors which influence the teaching - learning, to identify and address the mentioned problems, by proposing personal tutoring in the University as appropriate channel for commissioning practice of this project.

\section{KEYWORDS: Diversity - Learning difficulties - Basic Skills - Strategies - Teaching- Neuropsychology - Tutoring University.}

\section{Cómo citar el artículo:}

Lina $M^{\mathrm{a}}$ Tomás Pastor, Sonia $\mathbf{M}^{\mathrm{a}}$ Martínez Castro y $\mathbf{M}^{\mathrm{a}}$ José Lombarte Londres- (2013) EVALUACIÓN DE LAS ESTRATEGIAS DE APRENDIZAJE EN LA UNIVERSIDAD: UN PROYECTO NECESARIO ANTE UN ALUMNADO DIVERSO. Revista de Ciencias de la Comunicación e Información. (Diciembre 2013). Año XXVI (30), 59-75 ISSN: http://www.revistaccinformacion.net/ARTICULOS/2013\%20e-j\%20Bea/2013\%20e\%20Evaluaci\%C3\%B3n\%20de\%20las\%20estrategias\%20de\%20aprendizaje..\%20Tom \%C3\%A1s,\%20Mart\%C3\%ADnez\%20y\%20Lombarte\%20(UCAM).pdf

\section{INTRODUCCIÓN}

\subsection{Un alumnado universitario diverso}

Son varios los puntos de reflexión acerca del tipo de alumnado que tenemos en las aulas: la procedencia tanto profesional como académica, el perfil del alumno, y la diversidad. Hoy en día, la diversidad que encontramos en los estudiantes debe ser trabajada desde las aulas a través de una innovación docente y de calidad y una educación personalizada.

Los datos oficiales de la última edición de Datos y Cifras del Sistema Universitario español (SUE), servirán de guía para este estudio. Se trata de una plataforma conjunta del Ministerio de Educación, Cultura y Deporte, las Comunidades Autónomas y las Unlversidades, mediante la cual obtenemos datos tales como que el número de estudiantes mayores de 30 años aumenta, y el número de egresados cada vez es mayor, situándose la edad de éstos entre 31-40 años de edad. Sin duda, este dato puede deberse a los cursos de adaptación existentes entre las antiguas diplomaturas o ingenierías técnicas y los actuales Grados que se realizan en un buen número de Universidades.

La procedencia de alumnos en las aulas es variada: alumnos extranjeros, ingresos a través de ciclos formativos, mediante pruebas de acceso a la Universidad, o también 
acceso mediante otros títulos universitarios (caso de la UNED).

Otro dato que nos aporta esta plataforma es la formación de los padres, siendo un indicador importante, sobre todo si atendemos a los alumnos que acceden a los grados procedentes de PAU (Pruebas de acceso a la Universidad), o FP (Formación Profesional), donde la mayoría de sus padres poseen estudios superiores, siendo trabajadores de la administración y sector servicios. Sin duda, la mayoría de estudiantes que nos encontramos son mujeres, tanto de Grado como de Máster.

Para abordar la importancia de atender los valores en la educación, es necesario replantear el trabajo educativo desde todas las instancias, como la familia, administración pública, profesores, o los mass media. Pero este replanteamiento se debe de llevar a cabo sobre y desde el mismo sujeto de la educación que es la persona humana. De ahí la importancia de tutorizar en la Universidad, el significado que tiene la tutoría dentro del espacio Europeo de Educación Superior, cómo se realiza, las competencias del profesorado-tutor en la Universidad, el rol y funciones del tutor, que sin duda, es fundamental en toda institución donde se quieren atender y destacar los valores del alumno universitario, futuro ciudadano y profesional.

Como ya se ha dicho anteriormente, para hablar del tema que se plantea, la diversidad en las aulas, partimos como referencia de los datos obtenidos de una última edición (curso 2011-12) de Datos y Cifras del Sistema Universitario español (SUE). Es una plataforma conjunta que edita el Ministerio de Educación, Cultura y Deporte, las Comunidades Autónomas y las Universidades, que utiliza varios indicadores para realizar el estudio de la población universitaria española. Partimos de un dato que nos dice que la tasa neta de escolarización universitaria entre los 18 y 24 años en los últimos cursos está en continuo crecimiento, consecuencia del incremento del número de estudiantes universitarios y, a su vez, de la reducción de la misma población de esta edad.

También ha aumentado el número de estudiantes mayores de 30 años, y también el número de egresados, que cada vez es mayor, situándose éstos entre 31-40 años de edad. Sin duda, estas cifras tienen que ver con las posibilidades que se ofrecen para acceder a Grados superiores, como son los cursos de adaptación entre las antiguas diplomaturas o ingenierías técnicas y los actuales Grados que se realizan en un buen número de Universidades; también puede deberse a las propias circunstancias que en la sociedad de hoy en día tenemos, el papel que juega la crisis, donde mucha gente está en situación de paro laboral, o inactivos, y la decisión que toman es volver a las aulas para ampliar conocimientos y currículum.

Como podemos observar hoy en día en las aulas, la procedencia de los estudiantes es variada: alumnos extranjeros, alumnos provenientes de ciclos formativos, los que acceden mediante pruebas de acceso a la Universidad, o también a través de otros títulos universitarios.

Algo significativo y que ya conocemos es que la mayoría de estudiantes son mujeres, tanto de Grado como de Máster, y en todas las ramas, a excepción de las titulaciones técnicas; su presencia es mayoritaria en todos los niveles de formación universitaria situándose en un porcentaje del $59,1 \%$ como media. 
Como hemos dicho anteriormente, la presencia de estudiantes mayores de 30 años es cada vez más intensa entre los estudiantes universitarios de grado. Se ha incrementado en los últimos 5 años un 30\%, y un $82,8 \%$ en los últimos 10 años, hasta situarse de media en el $18,5 \%$ del total de estudiantes.

El número total de estudiantes extranjeros en estudios de grado, y máster del Sistema Universitario Español se incrementa en el curso 2011-2012. Se modifica la procedencia de los estudiantes extranjeros que cursan estudios de grado en España, de este modo, se reducen los estudiantes que proceden de Europa y, también los procedentes del Norte de África, pero mostrando el incremento en el número de estudiantes procedentes de América Latina y Caribe, junto con los procedentes de Asia y Oceanía .

Sin duda, hay cada vez más alumnos que se mueven por el interés de realizar en el extranjero parte de sus estudios, sin embargo, sigue siendo mayor el número de europeos que entran en España al de los españoles que salen. También aquí el papel de la mujer es mayoritario entre los alumnos Erasmus españoles. A nivel nacional existe el programa de movilidad interno Séneca, pero bien es cierto que no despierta el mismo interés y atractivo que el internacional, también porque el nivel de inversión es inferior al internacional.

Por lo tanto, vemos que debido a diferentes elementos o factores, la variedad en las aulas universitarias existe, pero también es cierto que a la hora de hablar de "variedad", pensamos en "diversidad", y no precisamente en ese término específico para algunos sujetos. Debemos pues, conocer el significado al que hace alusión y la relación con otros conceptos afines.

El concepto diversidad, según el diccionario de la Lengua Española, significa: variedad, semejanza, diferencia; pero en una segunda acepción se hace referencia a abundancia, concurso de varias cosas distintas (Buendía Eisman, 1990). La diversidad puede manifestarse en características propias de los individuos, y también en las características de los individuos como fruto del contexto. Será en esta última acepción en la cual nos fijaremos.

Educar en la diversidad supone, según Lizasoáin \& Peralta (1998), pensar en una escuela para todos, movernos en continuos tan opuestos como uniformidad / diversidad y homogeneidad / heterogeneidad. El término diversidad, es, por tanto, un constructo muy amplio que hace referencia a la diversidad cultural y no sólo a la diversidad física, incluyendo una gran variedad de posibilidades.

Realizar un análisis del crecimiento en el alumnado extranjero en los últimos años, exige ver el papel que tiene la escuela en la educación de los niños. La escuela constituye la institución clave a la hora de configurar las modalidades de inserción, socialización e inserción social de las nuevas generaciones. A través de las aulas, los hijos de las personas inmigrantes pueden acceder con más facilidad que las personas adultas e iniciar un proceso de cualificación formalizado. El sistema escolar está encargado de inculcar los valores básicos que definen la ciudadanía, es el vehículo de reproducción social, de este modo se integran a las poblaciones nuevas del país de residencia. 
Al hablar de diversidad en las aulas, se debe de hacer referencia también al papel que tiene la educación informal, pues es importante para la formación de todo ciudadano, siendo aquella que recibimos todos al vivir en sociedad, con constantes intercambios sociales y experiencias cotidianas, transmitiendo conocimientos, valores, actitudes, habilidades, etc, a lo largo de nuestra vida.

Dependiendo del perfil del estudiante universitario, se hace una comunidad diversa y enriquecedora, obligando al docente a conocer y demostrar su valía profesional en su trabajo con los valores dentro de la vida universitaria. Esto es lo que se exige y supone una educación en valores, porque a los alumnos se les debería de pedir un aprendizaje ético, y éste no es posible sin una educación en valores, en definitiva, una educación de calidad.

La formación de los profesionales de la docencia es fundamental, siendo uno de los papeles interesantes de la Universidad del siglo XXI de hoy en día. Los alumnos no solamente están llamados a obtener conocimientos para luego aplicarlos, sino también están llamados a ser ciudadanos responsables para contribuir en cada una de sus comunidades.

En el Informe a la UNESCO de la Comisión Internacional sobre la educación para el siglo $X X I$, presidida por Jacques Delors, se puede leer:

Para cumplir el conjunto de misiones que le son propias, la educación debe estructurarse en torno a cuatro aprendizajes fundamentales, que en el transcurso de la vida serán para cada persona, en cierto sentido, los pilares del conocimiento: aprender a conocer, ...; aprender a hacer, ...; aprender a vivir juntos, .; por último, aprender a ser, ..." (Delors, 1996, 95).

El informe de Jacques Delors titulado La educación encierra un tesoro (1996) tuvo como principal eje analizar de carácter profundo los cambios en la sociedad contemporánea, extrayendo de ahí el nuevo papel que tendría que tener la educación para el desarrollo sostenido de la humanidad.

Sería, pues, necesario volver a replantear seriamente el trabajo educativo (Gerstner, 1996), desde todas las instancias: familia, administración pública, profesores, y los demás agentes educativos, también desde los medios de comunicación: prensa, cine, televisión, radio, internet.

Pero este replanteamiento debería realizarse partiendo desde la persona humana, porque si se profundiza en la persona y su verdad, se podrá cimentar una nueva educación que supere las diferencias que pueden existir de raza, sexo, religión, y condición social, y que en algunos casos pueden llegar a destruir y dividir, haciendo del hombre enemigo del mismo hombre.

\subsection{Dificultades en el proceso de aprendizaje de los estudiantes universitarios}

Esta diversidad descrita que encontramos hoy en las aulas universitarias en edad, procedencia, como otras características individuales, nos obliga a repensar si las respuestas metodológicas que ofrecemos a nuestros estudiantes se adecuan a la renovación de las metodologías que se nos solicitaba con la incorporación al 
proceso de Bolonia iniciado en 1999. Esta adecuación de las metodologías pasa por conocer las características de un alumnado que se encuentra inmerso en una sociedad cada vez más incorporada a la era digital y tecnológica. Como comenta Hernández Pina, en el escenario del EEES "cabe hacerse la siguiente pregunta: ¿Cómo lograr que los estudiantes alcancen las competencias académicas mínimas necesarias y pongan en práctica un aprendizaje autónomo y autorregulado de acuerdo con las nuevas exigencias derivadas del proceso de Bolonia?" (2006, p.618). Pero además, esta adaptación, ya de por sí ardua, se ha ido encontrando con algunos escollos no previstos, derivados del propio proceso de aprendizaje de los estudiantes. Sabemos que

Aún así, asumiendo que estas preocupaciones educativas están consensuadas y generalizadas entre los educadores, nos enfrentamos con un conjunto de cuestiones relacionadas con las prácticas de la enseñanza-aprendizaje: ¿Por qué estos cambios no siempre ocurren? ¿Qué es preciso cambiar en el comportamiento de los alumnos para que ocurran? ¿Qué hacer de nuevo en las prácticas docentes? (...) (p. 573).

$Y$ es que nos estamos encontrando con frecuencia con alumnos que llegan a la Universidad con unas dificultades que tendrían que estar resueltas en etapas educativas anteriores. Se trata de herramientas y habilidades básicas no totalmente adquiridas en esas etapas precedentes, y que el profesorado no sabe hasta qué punto puede o debe atenderlas, hacerlas o no objeto de su propia docencia. No estamos hablando de estudiantes definidos como "alumnos con dificultades de aprendizaje", según la definición que ofrece la definición de dificultades de aprendizaje propuesta por la American Psychiatric Association y presentada en el Manual diagnóstico y estadístico de los trastornos mentales (2005), sino de alumnos con un desarrollo totalmente normal, pero que no se desenvuelven correctamente en la utilización de herramientas instrumentales básicas.

Hemos de indicar que el breve análisis que realizamos aquí de algunas de esas dificultades responde a la percepción que se tiene entre el profesorado, así como de la rica experiencia de atención individualizada que vamos teniendo gracias al servicio de tutorías personales de la Universidad Católica de Murcia (UCAM), y que es necesario afrontar en futuros trabajos, con la finalidad de conocer y definir de forma más precisa cuáles son estas dificultades, cómo abordarlas, y sus determinadas características dependiendo de los grupos y de los individuos a los que afecten.

Algunos de los problemas a los que nos referimos son los siguientes:

1. En primer lugar destacaríamos una falta de dominio de las reglas de ortografía: Es este uno de los aspectos que más nos suele llamar la atención, sobre todo en la corrección de exámenes de desarrollo o de trabajos escritos, incluso los elaborados a ordenador. Los alumnos presentan de forma generalizada una falta de conocimiento y de dominio del castellano escrito. Es posible que el uso de las tecnologías en una comunicación espontánea e instantánea como la que nos venimos habituando tenga que ver con el desarrollo de este problema, pero sería necesario un estudio más profundo sobre el tema. También podemos encontrar una 
explicación en la falta de hábito lector, que pondremos en la base de otras dificultades expuestas aquí. ¿Es la corrección ortográfica una tarea propia del profesor universitario? Pensamos que no.

2. Escasez de vocabulario, comprensión y velocidad lectora, provocado, entre otras cosas, por la falta de hábito lector. El poseer una buena comprensión y velocidad lectora se encuentra en la base de los aprendizajes, cualesquiera que sea el nivel o la etapa en la que se encuentren los estudiantes. Un aspecto que puede estar dificultando el proceso de estudio de los universitarios -y en muchos casos desconocido por ellos mismos-, es la falta de rapidez y fluidez en la lectura de libros, apuntes, etc. Los alumnos se cansan en su estudio, lo encuentran anodino y aburrido, sin caer en la cuenta de que el bajo nivel lector es en muchas ocasiones causante de estos problemas. También la falta de vocabulario y la reducción de palabras en su lenguaje comunicativo habitual, hace que se acentúen las dificultades a la hora de abordar unos contenidos de cierto nivel, como son los propios de la Universidad. Y es que el hábito lector enriquece el vocabulario, y el vocabulario enriquece el pensamiento.

3. Encontramos en los alumnos dificultades para hablar en público y expresar oralmente opiniones, así como de argumentar las propias ideas. El planteamiento de que nuestro sistema educativo español debería reforzar contenidos curriculares referentes a la exposición oral de trabajos, así como de las propias opiniones, no es algo nuevo. De hecho ya existen no pocas experiencias en centros de Primaria que utilizan estas metodologías. Sin embargo los debates, exposiciones, role - playing u otras metodologías que ayuden a que los estudiantes se habitúen a hablar delante de los compañeros, desde las edades más tempranas, debería convertirse en algo frecuente en las aulas de Primaria y Secundaria. Tendría que ser esta una habilidad ya desarrollada al incorporarse el estudiante a la Universidad, para que constituyera una herramienta básica de apoyo a la incorporación de otros conocimientos y no un objetivo propio de las enseñanzas universitarias.

4. Dificultades en la redacción y expresión escrita, a la hora de reelaborar y transferir información de forma personal. Unido a ello, existe una tendencia a la memorización mecánica de los conocimientos a la hora de estudiar. Este aspecto se ve reflejado de forma clara en la elaboración de los trabajos universitarios, donde se presentan evidentes dificultades para redactar de forma fluida, con una argumentación clara de lo que se quiere expresar. Esta dificultad se complementa con la tendencia a utilizar en exceso los contenidos de Internet, no como simple apoyo, sino con el famoso corto-pega, carente de una reelaboración adecuada de los contenidos. Enseñar a pensar con criterio y de forma crítica sí que es un objetivo propio de la etapa universitaria, pero para ello es necesario que los estudiantes, con la guía del profesor, suelten las amarras de la memoria mecánica -cuando ésta no sea totalmente necesaria- y se lancen a reflexionar sobre aquellos aprendizajes que van adquiriendo, a encontrar por ellos mismos los puntos de unión entre unas asignaturas y otras, a extraer conclusiones propias de una etapa de desarrollo de la madurez personal y de preparación profesional. En definitiva, uno de los objetivos de la Universidad es enseñar a los estudiantes a pensar, y a quitar los obstáculos que potencien esta tarea.

5. Asimismo, se da de forma cada vez más generalizada una propensión a la 
ansiedad y poca resistencia a la frustración y a la superación de dificultades. Se observa un descenso generalizado en la resiliencia de los estudiantes, considerando esta como la capacidad de resistencia a las dificultades e incluso salir fortalecido de ellas (Henderson, 2003). Se refleja un aumento en procesos de ansiedad en personas cada vez más jóvenes, a veces con pocas herramientas psicológicas y emocionales para hacer frente a las dificultades personales y de relación que se les presentan. Este aspecto queda reflejado de forma evidente a la hora de realizar los trabajos en equipo y en la gestión de los exámenes. Bolonia ha incrementado la elaboración de prácticas y trabajos grupales, siendo el trabajo en equipo una de las competencias genéricas a desarrollar en los alumnos. Parece que los conflictos normales de todo trabajo en colaboración se acentúan en jóvenes con muchos recursos emocionales y afectivos, pero en ocasiones poco entrenados para darles cauce y adecuarlos a las situaciones de la forma más conveniente. Tal vez habría que buscar algunas de estas causas en el proteccionismo familiar, en padres que, desde que los hijos son pequeños, se adelantan a solucionar las dificultades y siguen haciéndolo cuando los hijos crecen -hasta cuando llegan a la Universidad-. Ayudar por lo tanto a los alumnos a adquirir un adecuado equilibrio emocional, a gestionar correctamente su afectividad en función de las situaciones y circunstancias que está viviendo, es otra de las tareas dentro del marco de la atención personalizada, que la Universidad actual tiene delante de sí.

Estas dificultades nos abren a investigaciones presentes y futuras. Lo que hace unos años eran considerados como aspectos puntuales en ciertos alumnos, se presenta en la actualidad de forma más difundida, debido entre otros motivos a la apertura de las aulas a una gran diversidad de alumnado. Como decimos, los problemas que hoy se presentan han pasado de ser circunstanciales -mal comportamiento de los estudiantes, pocas ganas de estudiar, desequilibrios emocionales puntuales...- a dificultades de base del aprendizaje -problemas de comprensión, expresión, reelaboración lecto-escritora, inmadurez personal y afectiva...-, lo que provoca el desconcierto entre los profesores y su resistencia -justificada, en muchas ocasiones- a atender problemáticas más propias de otros niveles educativos.

Sin embargo, puede haber tal vez otro punto de vista que tengamos que tener en cuenta, y es saber si nos encontramos ante un nuevo paradigma, unas nuevas formas de adquirir los aprendizajes. Las nuevas demandas sociales, las presentes y futuras formas de trabajo y de comunicación, el aprender con una tecnología cada vez más avanzada y todo el abanico de nuevas posibilidades que se van abriendo a nuestro ojos, nos hace plantearnos si es el sistema y el currículo el que ha de adaptarse a las nuevas formas de enfrentarse a la realidad que van adquiriendo los niños y jóvenes. Así lo afirma en 2004 Siemens, creador de la corriente conectivista, al referirse a los procesos de aprendizaje en los entornos digitales: "el acceso a lo que se necesita es más importante que lo que el aprendiz posee actualmente" (2004, p. 8).

¿Va a sustituir la importancia del saber acceder a los conocimientos a los propios conocimientos, especialmente a aquellos que consideramos básicos? Por ellos es necesaria más que nunca una clarificación de en qué consisten esas nuevas formas de aprendizaje, qué conocimientos elementales deberán dominar los estudiantes sin poder transigir sobre ellos, y qué conocimientos sufrirán las transformaciones de los nuevos modelos. 


\subsection{La neuropsicología: una respuesta para la aplicación de estrategias de aprendizaje}

El concepto de aprendizaje es tan amplio como el número de teorías que han intentado explicarlo. Desde las teorías constructivistas de Piaget que consideran el aprendizaje como un proceso de adaptación al medio que nos permite modificar nuestra conducta como resultado de la práctica, hasta las teorías en las que se contempla el aprendizaje como un proceso que recoge información, la procesa y produce una respuesta.

Teniendo en cuenta todas estas teorías, podríamos determinar que el aprendizaje nos permite adquirir nuevos conocimientos de forma progresiva a través de la propia experiencia, adaptando los nuevos descubrimientos con aquello que ya conocemos, dando de esa manera significado a esas experiencias que tiene como respuesta la modificación de nuestra conducta.

Si nos ceñimos al ámbito educativo, el proceso de aprendizaje en palabras de Beltrán (2003)"se utiliza para significar la cadena general de macro- actividades u operaciones mentales implicadas en el acto de aprender" (pg.56)

Estas operaciones mentales, desde el punto de vista de la Neuropsicología, vienen marcadas por el funcionamiento de una serie de sistemas neuro-evolutivos cuya combinación permite un sinfín de posibilidades. Estos sistemas se pueden desarrollar y pueden determinar la capacidad de aprender de un alumno. El funcionamiento y la ejercitación de dichos sistemas, pueden ser, sin duda, la clave para un rendimiento académico eficaz y satisfactorio.

La Neuropsicología es la ciencia que estudia de las funciones cerebrales y su relación con el comportamiento, su aportación en el campo de la salud ha sido determinante para el tratamiento de muchas enfermedades con déficits neurológicos.

La aplicación de todos los descubrimientos sobre el funcionamiento cerebral y su plasticidad en el campo de la atención temprana también ha dado grandes resultados. Otro de sus retos es el estudio de las distintas áreas cerebrales, su funcionamiento y su aplicación en el campo de la educación. La localización e identificación de los distintos procesos de aprendizaje que tienen lugar cuando se trata de adquirir, elaborar, almacenar, recuperar y transferir una información, nos pueden llevar a planteamientos distintos para introducir al alumno en el uso de estrategias de aprendizaje de una forma más individualizada, más acorde a sus propias características.

La diversidad ante el proceso de enseñanza- aprendizaje se pone de manifiesto en las palabras del Doctor Levine (2003):"Cada persona está dotada de un conjunto de circuitos innatos y muy complejos que crean innumerables vías y bifurcaciones que representan opciones y obstáculos"(p.26).

En su tesis sobre el proceso de aprendizaje, describe ocho sistemas neuroevolutivos cuya funcionalidad es la base para la adquisición de ciertas destrezas y habilidades. 
1. Sistema de control de la atención es el que permite al estudiante mantener la concentración.

2. Sistema de la memoria

3. Sistema lingüístico necesario para la comprensión lectora, la expresión oral y escrita, el aprendizaje de idiomas.

4. Sistema de ordenación espacial imprescindible para la organización.

5. Sistema de ordenación secuencial es importante para tratar la información y para la comprensión y cálculo del tiempo.

6. Sistema motor que dirige las funciones tanto de la motricidad gruesa como en la motricidad fina.

7. Sistema del pensamiento de orden superior relacionado con el razonamiento lógico, la capacidad para resolver problemas incluyendo también el pensamiento crítico y el pensamiento creativo.

8. Sistema de pensamiento social es el que ayuda al estudiante a establecer y mantener relaciones sociales de forma adecuada.

Todos estos sistemas trabajan conjuntamente, para obtener buenos resultados y deben ser ejercitados en todas las etapas de la vida académica del alumno. Esta ejercitación requiere el uso de procedimientos intencionales a los que llamamos estrategias, entendiendo por estrategia: "Las operaciones mentales internas que se activan para facilitar la adquisición, el almacenamiento y la utilización de la información." (Beltrán, 1998)

La ejercitación y desarrollo de estrategias variará en función de las necesidades y características de cada alumno, desde aquéllas que integran los procesos de adquisición y selección de la información para ejercicio de los sistemas de atención hasta las de procesamiento y organización de la información en la que influyen los sistemas de ordenación espacial y secuencial, por ejemplo.

Desde el punto de vista de la Neuropsicología, las conexiones neuronales se establecen mediante la realización de distintas actividades, desde las más básicas de tipo sensorial y motor, hasta las más complejas, hablar, leer, escribir, pensar; cuando estas actividades se producen en condiciones de intensidad, frecuencia y duración suficiente, se pone en marcha el proceso de aprendizaje, un proceso gradual que se prolonga a lo largo de toda la vida.

Son muchos los aspectos que pueden influir en el desarrollo de las funciones que desarrollan estos sistemas definidos anteriormente: factores genéticos, familia, cultura, amistades, salud, emociones y por supuesto, la experiencia educativa. Todos estos factores nos van a permitir también trazar un perfil del alumno, en cuanto a sus características personales, su estilo de aprendizaje y sus necesidades para obtener un rendimiento eficaz.

Por lo tanto, el análisis descrito nos lanza a la presentación de un proyecto de intervención en el ámbito universitario, partiendo de las investigaciones en Neuropsicología, el cual pretende aportar luces al trabajo universitario, para poder comprender y afrontar los grandes cambios que se avecinan, ya no solo externos o circunstanciales al propio alumno, sino también los relativos a los procesos internos de aprendizaje de los estudiantes, así como la adaptación de los procesos de enseñanza del profesorado a las nuevas formas de aprender de los alumnos. E 
cauce para llevar adelante el proyecto será la tutoría universitaria. Por lo tanto, nos encontramos ante un reto en el que todas las partes se encuentran implicadas, preocupadas por la mejora continua de los procesos de enseñanza-aprendizaje, no sólo en las etapas de Primaria y Secundaria sino, - y esto es en lo que a nosotros nos atañe-, también en la Universidad.

\section{OBJETIVOS}

1. Evaluar a los estudiantes de nuevo ingreso en el uso de estrategias de aprendizaje.

2. Relacionar el uso de estrategias de aprendizaje con variables indicadoras de la diversidad del alumnado: sexo, edad y procedencia.

3. Valorar la percepción del alumno de su propio rendimiento académico y sus habilidades de aprendizaje.

4. Valorar la percepción del profesorado sobre sus propias estrategias metodológicas.

5. Elaborar programas de intervención en la mejora de Estrategias y habilidades de Aprendizaje.

\section{METODOLOGÍA}

Este trabajo de investigación se plantea en el plazo de dos años académicos y está dirigido a alumnos de nuevo ingreso.

En una primera fase se llevará a cabo la valoración inicial de los alumnos en cuanto a la percepción que tienen sobre su propio proceso de aprendizaje, sus hábitos de estudio y sus características personales.

Así como una valoración de la percepción del profesorado sobre su conocimiento de estrategias de aprendizaje y la introducción de las mismas en su materia de conocimiento.

Esta valoración responde a una metodología cualitativa sujeta a un modelo descriptivo en el que se analizan las características de los alumnos sujetos de estudio.

Por otra parte, para establecer las relaciones entre diversidad del alumnado y utilización de estrategias de aprendizaje, se utilizará un análisis de correlaciones.

\section{Instrumentos de medida:}

- Cuestionario de Autopercepción del rendimiento académico y habilidades de aprendizaje.

- Cuestionario de Autoevaluación de estrategias metodológicas del profesorado.

- Escala abreviada para universitarios ACRA (Jesús de la Fuente y Fernando Justicia)

La Escala Acra ( Román y Gallego, 1994) permite evaluar las estrategias de aprendizaje de un estudiante que se corresponden con Adquisición, Codificación, Recuperación y Apoyo de la información. Posteriormente, en 2003, Jesús de la 
Fuente y colaboradores elaboran la Escala Acra-abreviada para alumnos universitarios, resumiendo a 44 ítems para valorar tres dimensiones:

Dimensión I. Estrategias cognitivas y de control del aprendizaje.

Dimensión II Estrategias de Apoyo al aprendizaje.

Dimensión III. Hábitos de estudio.

\section{Muestra:}

Alumnos de nuevo ingreso de los Grados de Educación Infantil y Primaria de la Universidad Católica San Antonio (UCAM).El proyecto comenzará en el curso 20142015 y se prolongará durante dos años.

\section{CONCLUSIONES Y DISCUSIÓN}

Siempre que ha existido un buen maestro, ha existido esa preocupación por la calidad educativa, de la misma forma que si existe un buen alumno, o unos buenos padres, o familia, ya que la calidad se refleja en las acciones permitiendo distinguir lo que está bien de lo que está mal.

Por tanto, la calidad está en lo que mismo hacemos, en la esencia misma de nuestras acciones y actividad diaria. Se debe de hacer todo con buena intención y conocimiento de lo que se debe de hacer en relación a la educación y la persona. De este modo, llegamos a la definición de calidad educativa que García Hoz nos aporta:"Se podría definir la calidad educativa como el modo de ser de la educación que reúne las características de integridad, coherencia y eficacia" (García hoz, 1982, p.2). La integridad y la coherencia se refieren al ser mismo de la educación, mientras que la eficacia se refiere a la actividad de la educación. "El ser y el hacer de algo se hallan afectados directamente por su calidad” (García Hoz, 1982, p. 3)

La integridad, la coherencia, la eficacia, son tres elementos que deben de estar presentes en toda acción educativa desarrollada con criterios de calidad.

El Espacio Europeo de Educación Superior es un ambicioso y complejo plan que han puesto en marcha los países del viejo continente para favorecer en materia de educación la convergencia europea. En el se habla de la figura del tutor en la Universidad, pero nos podemos preguntar si el análisis y la implementación que la función tiene esta figura es relevante en el marco que nos planteamos.

Se ha acrecentado el interés en torno a la tutoría en la formación superior del estudiante radica en el intento de dar respuesta a las nuevas necesidades de la institución universitaria y de los estudiantes que acceden a ella. La evolución de las universidades, desde una perspectiva de minorías a una universidad de masas, obliga a mejorar la atención de los discentes, en el sentido de introducir actuaciones dirigidas hacia un trato o relación más personal e individualizada. Por tanto, parece necesario asumir el principio de la diversidad, tanto en los objetivos de formación como en las características de sus protagonistas (Rodríguez, 2004). 
Por otra parte, los retos que se derivan de la creación de este espacio Europeo de Educación Superior, exige el que aparezca un sistema de apoyo al alumno, permitiéndole convertirse en el verdadero protagonista del proceso de enseñanza aprendizaje.

La figura del tutor y su papel coordinada dentro de un plan de acción debe de ser el elemento clave de este sistema de apoyo. Para ello se hace imprescindible la asunción de ciertos roles y competencias por parte de los tutores que guían a sus estudiantes.

Requiere de la adquisición por parte del profesorado de una serie de competencias y funciones relacionadas con el quehacer profesional del profesorado tutor.

Debe de reunir el tutor un conjunto de conocimientos y habilidades que los sujetos necesitamos para desarrollar algún tipo de actividad (Zabalza, 2006), tratándose de un trabajo complejo.

La competencia tutorial forma parte sustancial del perfil profesional del docente universitario. "defensor, guía, protector" son algunas de las acepciones atribuidas al docente y también al tutor. El profesorado, tal y como acabamos de mencionar comparte estos aspectos: es el profesor que guía el desarrollo personal y la formación del estudiante; es la persona fuerte y experimentada que defiende al tutorando de la novedad y las incertidumbres del inicio de cualquier proceso profesional; es el orientador técnicamente competente capaz de orientar hacia el ejercicio profesional al que el tutorando se incorpora; y es la persona prudente y amiga que sabrá también defenderla de las presiones y conflictos a los que su propia inexperiencia le podría conducir con excesiva facilidad (Brigall, 2000).

Mediante la tutoría se puede ayudar a romper el anonimato, la soledad, que aparece en las clases masificadas, y puede también influir en los diversos aspectos que se relacionan con el desarrollo personal de los estudiantes. Como se observa, existe un hilo conductor entre docencia, tutoría y orientación. El profesorado tiene una función docente e investigadora, pero además una función tutorial. Todo educador, por el sólo hecho de serlo, asumirá una función orientadora, en donde el trabajo de y con los valores ha de ser importante y fundamental para esa educación de calidad e innovadora.

Durante estos últimos años, el contacto directo y personal con alumnos universitarios de nuevo ingreso de distintas titulaciones, dentro del programa de tutorías personales de la UCAM, nos ha permitido tener una visión generalizada de los potenciales y los déficits de aprendizaje con los que llegan los alumnos a la universidad y constatar un hecho, los estudiantes con éxito difieren de los estudiantes con menos éxito en que conocen y usan estrategias de aprendizaje (González y Díaz, 2004). En este sentido, las estrategias ofrecen a la educación un tipo de tecnología especialmente útil para la intervención educativa (Beltrán, 2003).

Si hacemos referencia a alguna de las investigaciones realizadas con alumnos universitarios en distintas Universidades españolas, en relación al uso de estrategias y su repercusión en el rendimiento académico, podemos concluir que "se han obtenido correlaciones positivas y sugieren una mejora en los procesos de 
aprendizaje de los alumnos que participaron en el programa de promoción de procesos y estrategias de aprendizaje "(Rosario, Núñez y González Pineda, 2007).

Diagnosticar las estrategias de aprendizaje de un alumno universitario puede parecer innecesario, sin embargo la realidad se impone. Los niveles en muchas de las destrezas necesarias para que se produzca el aprendizaje son más bajos de lo deseable, lo cual afecta directamente a la funcionalidad de los sistemas de orden superior, entre los que se encuentran: la capacidad para resolver problemas, razonar lógicamente, adquirir y aplicar conceptos, comprender cómo y cuándo se aplican las reglas y captar el sentido de una idea compleja, lo que incluye el pensamiento crítico y el pensamiento creativo.

Este reto, sin embargo, se puede afrontar a través de entrevistas individuales como las que se realizan en la Tutoría personal, o bien de forma generalizada, con la utilización de cuestionarios estandarizados que nos permitan obtener un perfil del alumno.

Es obvio que el cambio social que ha ocurrido en los últimos años ha sido tan rápido que apenas hemos tenido tiempo de adaptar la metodología educativa a las necesidades de nuestros alumnos.

Por otra parte, podría ocurrir que la influencia de las nuevas tecnologías y las nuevas formas de comunicación hayan influido en los distintas Funciones neuroevolutivas, potenciando ciertas destrezas en detrimento de otras.

Existen algunos métodos educativos que tienen en cuenta estas bases neuropsicológicas para mejorar el aprendizaje, entre los que cabe destacar, el programa $\mathrm{ADI}$, es un programa de Apoyo y Desarrollo de la Inteligencia propuesto por profesionales del Instituto de Neuropsicología y Educación de Villanueva de la Cañada,

El trabajo en la primera fase se corresponde con los niveles de educación infantil y primaria, mientras que la segunda fase se aplica en alumnos de secundaria y bachillerato.

La gran importancia de esta segunda fase es que se trabaja con el alumno en el autoconocimiento, autocontrol, autoevaluación, es decir todas aquellas estrategias que tienen relación con la autorregulación que son en realidad las pueden mejorar su rendimiento académico en este nivel educativo y por tanto, conseguir que su proceso de aprendizaje sea más efectivo, permitiendo mejorar el binomio esfuerzoeficacia.

Hasta el momento, todos los estudios relacionados con la implantación del programa ADI en colegios, han demostrado la eficacia de este método en la mejora del rendimiento académico de los alumnos, lo que nos plantea como posible objetivo la formación del profesorado para el desarrollo de metodologías que favorezcan el uso de estrategias de aprendizaje y que promuevan el pensamiento crítico y creativo, facilitando así la adaptación del alumno a las exigencias de un nivel de estudios universitario.

Un aprendizaje que sea eficaz requiere una funcionalidad adecuada entre lo que se 
aprende y la aplicación de lo aprendido, es decir, permite al alumno elaborar y cuestionar lo que se le enseña, examinar la nueva información asociarla con las informaciones que ya posee y sobre todo, generar nuevas estructuras de conocimiento.

Todas estas funciones mentales son las que desempeñan un papel fundamental en el rendimiento académico.

En realidad, todo apunta hay que darle un papel más relevante al alumno sin caer en la tentación de bajar los niveles de exigencia sino propiciando el aprendizaje, a todos los niveles, incorporando distintos métodos de enseñanza que respondan al resultado de investigaciones científicas.

\section{REFERENCIAS}

Beltrán, J. (1998). Procesos, Estrategias y Técnicas de Aprendizaje. Madrid: Síntesis.

Beltrán, J. (2003). Estrategias de Aprendizaje. Revista de Educación, núm.332, pp. 55-73.

Buendía L. (1990). Evaluación y atención a la diversidad. En Salmerón, H. (Coord.). Evaluación educativa. Teoría, metodología y aplicaciones en áreas de conocimiento. Granada: Grupo Editorial Universitario.

Delors, J. (1996). La educación encierra un tesoro. Informe a la UNESCO de la Comisión Internacional para el siglo XXI. Madrid: Santillana.

De la Fuente, J (2011). Escala de estrategias de aprendizaje ACRA-Abreviada para alumnos universitarios, en Revista Electrónica de investigación Psicoeducativa y Psicopedagógica, nำ (2) pp. 140-158.

García Hoz, V. (1982). Calidad de la Educación, trabajo y libertad. Madrid: Dossat.

García, A.J. y Troyano, Y. (2009). El espacio europeo de Educación Superior y la figura del profesor tutor en la Universidad, en REDU Revista de Docencia, no 3, pp. 33.

Gerstner, L. et al. (1996). Reinventando la educación. Barcelona: Paidós.

González, D .y Díaz,Y.M.(2006). La importancia de promover en el aula estrategias de aprendizaje para elevar el nivel académico en los estudiantes de Psicología, en Revista Iberoamericana de educación, Vol 40 no 1. Recuperado de:

http://dialnet.unirioja.es/servlet/articulo?codigo=2098498

Henderson, E. (2003). Nuevas tendencias en resiliencia. En Resiliencia, descubriendo las propias fortalezas. Barcelona: Paidós.

Hernández Pina, F. et al. (2006). Promoción del aprendizaje estratégico y competencias de aprendizaje en estudiantes de primero de Universidad: 
Evaluación de una intervención, en Revista de Investigación Educativa, 24(2), pp. 615-631.

Hernández Pina et al. (2010). Impacto de un programa de autorregulación del aprendizaje en estudiantes de Grado, en Revista de Educación, 353, Septiembre- Diciembre, pp. 571-588.

Levine, M. (2003). Mentes diferentes, aprendizajes diferentes. Barcelona: Paidós.

Lizasoáin, O. y Peralta López, F. (1998). Atención a la diversidad. En R, Bisquerra Alzina (Coord.). Modelos de Orientación e Intervención Psicopedagógica, pp. 273-280. Barcelona: Praxis Universidad.

Mason, A. y Mason, M. (2005). Estudiantes universitarios con dificultades de aprendizaje, en PhDb Pediatr Clin, 52, pp. 61 - 70.

Rodríguez, S. (Coord.) (2004). Manual de tutoría universitaria. Recursos para la acción. Barcelona: Octaedro (ICE-UB).

Rosario, P. Núñez, J. C. y González Pineda, J. ( 2007). Eficacia de un programa instruccional para la mejora de procesos y estrategias de aprendizaje en la enseñanza superior, en Psicothema, Vol.19 no 3, pp.422-427.

Siemens, G. (2004). Conectivismo. Una teoría de aprendizaje para la era digital. Recuperado de: http://www.slideshare.net/lepirex/siemens2004conectivismopdf-presentation 
EVALUACIÓN DE LAS ESTRATEGIAS DE APRENDIZAJE EN LA UNIVERSIDAD:

UN PROYECTO NECESARIO ANTE UN ALUMNADO DIVERSO 
